\title{
Separation Properties of Countable Infinite Iterative Function System Based on the Discrete Element Method
}

\author{
Xiaochun Tang, Wei Zhang, Meili Dai \\ Rizhao Polytechnic, Rizhao, 276826, China \\ xiaochun tang@yeah.net
}

\begin{abstract}
Keywords: Discrete element method; Countable; Geometric-fractal; MATLAB software; Approximation curve; Iterative function

Abstract. The current research on finite iterated function system has been quite mature, and infinite iterated function system satisfying the open set condition also made some important results. This paper introduces the discrete element theory and fractal geometry theory into the separation of countable infinite iterated function system calculation, and sets up a discrete element fractal equation of infinite iterated function. In order to verify the effectiveness and reliability of discrete element model and algorithm, this paper uses MATLAB of matrix sum function to calculation software to test of the model, and obtains the discrete approximation curve of countable infinite iterated function by numerical simulation. In order to test the universality of algorithm, this paper calculates the iteration speed of three algorithms, we find that the three algorithms can achieve good function iterative discrete approximation, and have good effect fractal of genetic algorithm. It provides the theoretical basis for the research of countable infinite iterated function system.
\end{abstract}

\section{Introduction}

Discrete element method is a numerical simulation method to solve discontinuous media problem, its application in the calculation of iterative function can realize the fractal of iterative function. MATLAB is commercial mathematical software produced by MathWorks Company, which is used for high-level technical computing language and interactive environment of algorithm development, data visualization, data analysis and numerical computing, including MATLAB and Simulink. This paper uses the discrete element theory to establish the mathematical model of countable infinite iterated function separation, and uses MATLAB software to realize the function of programming process $[1,2]$. Finally, through the Simulink of MATLAB toolbox we experiment algorithm on the mathematical model, and obtain the separation characteristics of countable infinite iterated function. It provides a new computer method for infinite iterated function system.

\section{MATLAB Discrete Element Method Overview of Infinite Iterative Function}

The MATLAB matrix sum function calculation is the most commonly used software. It has powerful matrix calculation function and supports programmable function iterative calculation [3]. Numerical simulation module Simulink module has many ready-made functions to select, which is a very efficient mathematical tool. This paper chooses Simulink iterative function calculation of MATLAB toolbox to program the countable infinite iterated function programming and solve discrete solution, and gets function iterative solution.

Figure 1 shows the solving process of countable infinite iterated function system [4]. Firstly it uses the discrete element theory to do discrete treatment on the function, by using the function partition divides the function into discrete element form, then solves function by iterative method, finally obtains function discrete solution. 


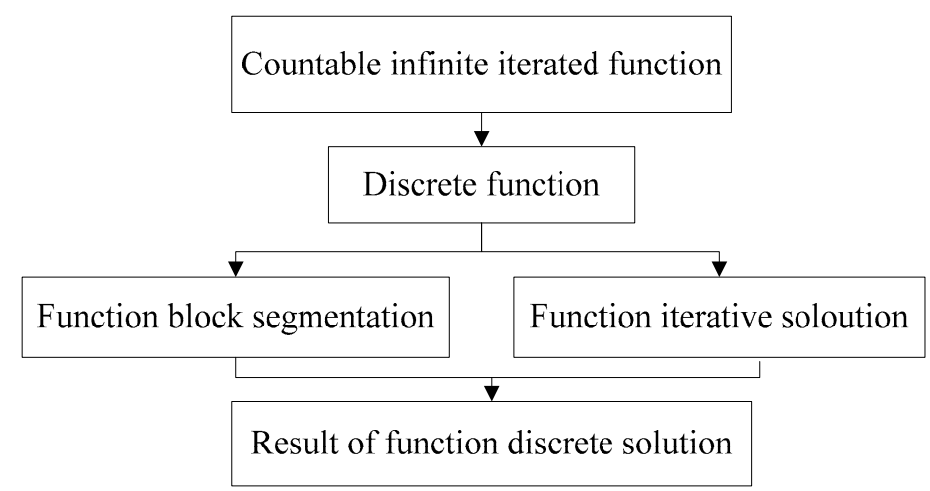

Fig. 1 The flow chart of prior structure compressed sensing image processing

\section{The Discrete Mathematics Model and Algorithm Design of Infinite Iterated Function}

Iterative function is divided into finite iterative and non iterative. This paper uses the countable infinite iterated function, so in the iterative calculations it needs to assume that the iterations are $n$ [5]. For the function $f(x), f^{(1)}(x)=f(x), f^{(2)}(x)=f\left(f^{(1)}(x)\right), \ldots, f^{(n)}(x)=f\left(f^{(n-1)}(x)\right)$, $(n \geq 2, n \in N) . f^{(n)}(x)$ is called $n$ iteration of $f(x)$.

If $a \neq 1, f(x)=a x+b, x_{0}$ is fixed point of $f(x)$, for the positive integer of $n$, we can get:

$f^{(n)}(x)=a^{n}\left(x-x_{0}\right)+x_{0}$.

So we can get $f(x)=a x+b, x_{0}=a x_{0}+b$. Two formulas subtract:

$f(x)-x_{0}=a\left(x-x_{0}\right)$.

When $n=1$, from the formula (1) we know the conclusion is right. When $n=k$, the conclusion is also right. When $n=k+1$,

$$
\begin{aligned}
& f^{(k+1)}(x)=f\left(f^{(k)}(x)\right)=a\left(f^{(k)}(x)-x_{0}\right)+x_{0} \\
& =a\left(a^{k}\left(x-x_{0}\right)+x_{0}-x_{0}\right)+x_{0} \\
& =a^{k+1}\left(x-x_{0}\right)+x_{0}
\end{aligned}
$$

When $n=k+1$, the conclusion is also right. By the inductive principle, we know the conclusion is right. In order to realize the function fractal, we first introduce the discrete element theory, coupled with the discrete initial data.

$$
X^{(0)}=\left\{X^{(0)}\left(t_{1}\right), X^{(0)}\left(t_{2}\right), \ldots X^{(0)}\left(t_{n}\right)\right\} .
$$

Doing one discrete on $X^{(0)}$

$$
X^{(1)}=\left\{X^{(0)}\left(t_{1}\right), \sum_{k=1}^{2} X^{(0)}\left(t_{k}\right), \ldots \sum_{k=1}^{n} X^{(0)}\left(t_{k}\right)\right\} \text {. }
$$

Doing $m$ discrete on $X^{(0)}$.c

$$
X^{(m)}\left(t_{i}\right)=\sum_{k=1}^{i} X^{(m-1)}\left(t_{k}\right) \text {. }
$$

In the process of calculation, we can use the double logarithmic curve to establish the relationship between $N(\varepsilon)$ and $\varepsilon$. Through the fitting of line slope, it can achieve fractal of iterative function [6]. And the first order fractal dimension function is:

$$
D 1_{i, i+1}=\frac{\ln \left(S 1_{i+1} / S 1_{i}\right)}{\ln \left(k_{i} / k_{i+1}\right)} .
$$

Two order fractal dimension is:

$$
D 2_{i, i+1}=\frac{\ln \left(S 2_{i+1} / S 2_{i}\right)}{\ln \left(k_{i} / k_{i+1}\right)} \text {. }
$$

$I$ order fractal dimension is: 


$$
D I_{i, i+1}=\frac{\ln \left(S I_{i+1} / S I_{i}\right)}{\ln \left(k_{i} / k_{i+1}\right)} .
$$

In order to realize the iterative and fractal dimension of infinite iterated function, this paper uses MATLAB software to program the countable infinite iterative function; the main program used is as follows:

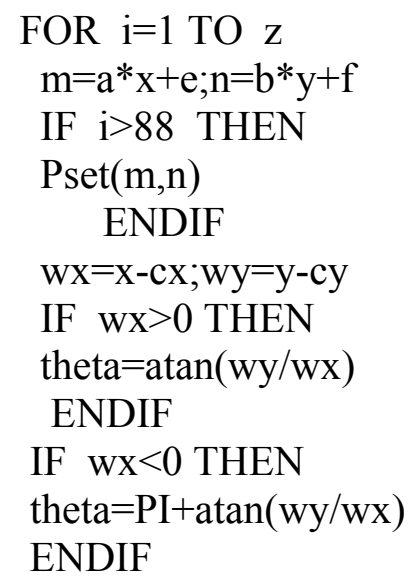

\section{Ddiscrete Calculation Numerical Simulation of Countable Infinite Iterative Function}

In order to verify the validity and reliability of mathematics model and algorithm for infinite iterative function designed in second part, this paper uses professional simulation software MATLAB to calculate the countable infinite iterated function system. Compared to the previous version, this version can use the effective profile, 10 functional $C$ code generation and 11 functional GPU acceleration, which can achieve image separation to make the separation of countable infinite iterative function system calculation more quickly.

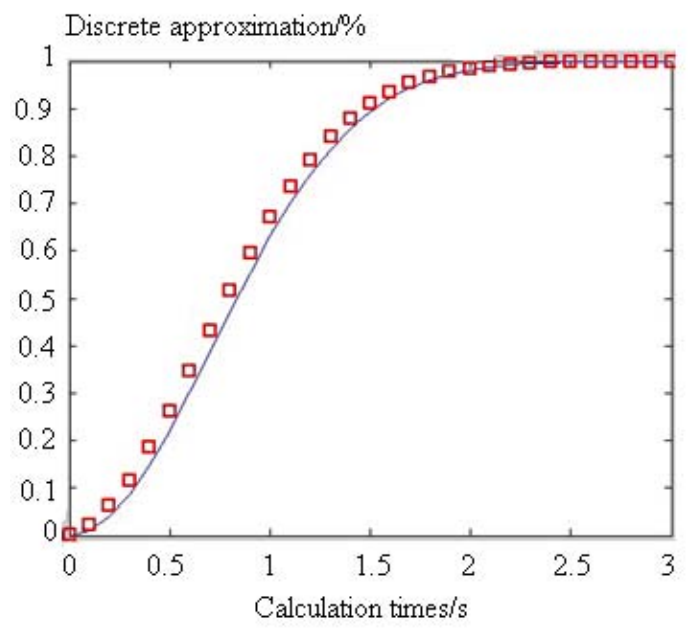

Fig. 2 Discrete element iterative comparison calculation process

Figure 2 shows the curve changing with time using MATLAB iterative calculation. From the chart we can see, in the initial stage, the approximation degree is close to 0 , with the increase number of iterations, the discrete approximation degree is more and more high, finally close to 1 , which proves the validity and reliability of the algorithm [7]. When applying it in the multidimensional countable infinite iterative function system, we obtain the results as shown in Figure 3. 


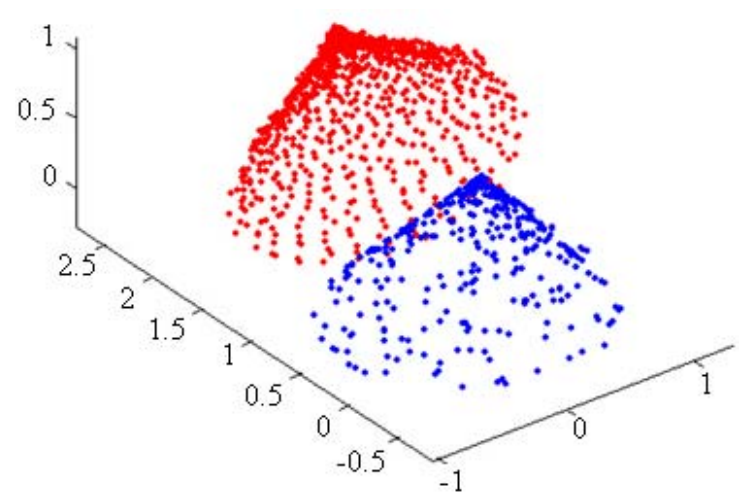

Fig. 3 Discrete process of iterative function system

Figure 3 shows the separation process on iterated function systems by using MATLAB toolbox Simulink. From the chart we can see, in the 3D distribution of the coordinate space, discrete element can effectively decompose function [8]. Through the function of fractal, we can extract discrete function with a certain separation characteristics.

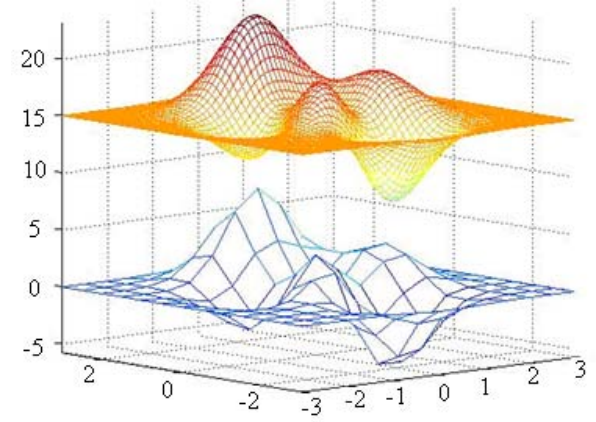

Fig. 4 Iterative separation results of discrete element function

Figure 5 shows the separation results of countable infinite function obtained by discrete element method. Using discrete element theory, MATLAB will discrete the function into unit to solve function matrix in each unit, so as to realize the separation characteristic function.

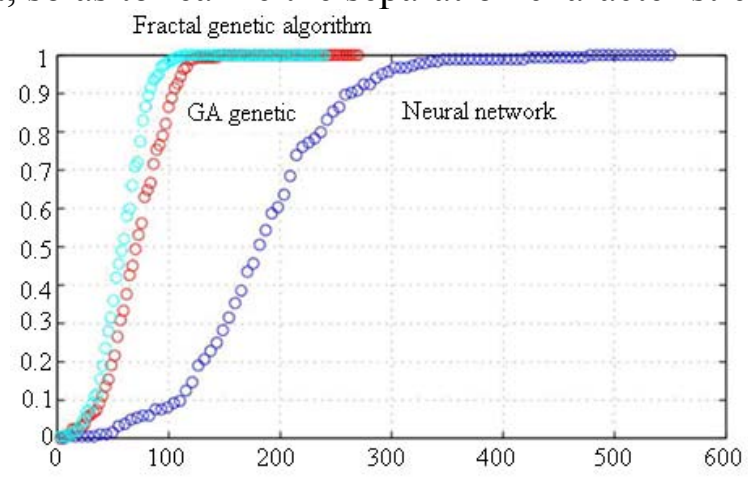

Fig. 5 Infinite iterative function discrete results for different algorithms

Figure 5 shows the approximation process curve of countable infinite iterated function separation using different algorithms [9]. From the chart we can see, the speed of different algorithms function separation calculation is different, the computing task can be completed in 400 step iterative calculation. The calculation results parameters are as shown in Table 1.

Table 1. Infinite iterative discrete results with different algorithms

\begin{tabular}{|c|c|c|}
\hline Algorithm & Iteration number & Discrete approximation degree \\
\hline Neural network & 328 & 0.992 \\
\hline GA genetic algorithm & 129 & 0.996 \\
\hline Fractal genetic algorithm & 102 & 0.998 \\
\hline
\end{tabular}

Table 1 shows the infinite iterative discrete results with different algorithms. From the table we can see that the discrete approximation degree of three kinds of algorithms are relatively high, the 
iterative steps of fractal genetic algorithm require the least number, with high calculation fast, which is an efficient iterative algorithm.

\section{Summary}

This paper establishes the mathematical model of countable infinite iterative function system, and does discrete treatment on the equation using the discrete element form, and gets the fractal structure of infinite iterated function system. By means of $n$ fractal it realizes the infinite iteration calculation. We use MATLAB software to program the solution procedure of countable infinite iteration, and get the separation effect and discrete approximation curve of infinite iterated function by numerical simulation. Through the solving of countable infinite iterated function, we get the function separation process and results. From three different algorithms comparison analysis, the speed of fractal algorithm designed in this paper is fastest, with less number of iterations and relatively good performance.

\section{References}

[1] Deng Xiaoyan, Chen Xiaokun, Li Zhi. Construction algorithm and its operation properties of a class of Hermite fractal interpolation function [J]. College mathematics, 2011, 25(4): 45-48.

[2] Peng Tao, Feng Zhigang. Fractal interpolation function and its dimension with discontinuous points [J]. Journal of Xinyang Normal University (NATURAL SCIENCE EDITION), 2012, 21(4): 23-26.

[3] Fan Zhaolei, Wang Hongyong. The error analysis of two variables fractal interpolation function based on parameter perturbation [J]. Journal of Anhui University (NATURAL SCIENCE EDITION), 2012, 34(4): 101-104.

[4] Qian Xiaoyong, Peng Tao, Feng Zhigang. The maximum problem of a kind of fractal interpolation function [J]. College mathematics, 2012, 26(2): 78-82.

[5] Wang Hongyong, Ma Li. Error analysis of fractal interpolation function based on the variation of vertical scaling factors [J]. Journal of Xiamen University (NATURAL SCIENCE EDITION), 2011, 48(5): 18-22.

[6] Feng Zhigang Pang Xinxing. Fractal interpolation function and the vertical compression factor [J]. Journal of Lanzhou University of Technology, 2012, 36(3): 34-38.

[7] Zhou Aoying, Jin Cheqing, Wang Guoren, Li Jianzhong. The uncertainty of data management technology [J]. Journal of computer, 2012, 32(1):56-58.

[8] Dai Dongbo, Zhao Gang, Sun Shengli. Effective clustering algorithm based on probabilistic data stream [J]. Journal of software, 2013, 20(5): 1313-1328.

[9] Zong Yu, Li Mingchu, Jiang He. Approximate backbone guided reduction algorithm for clustering [J]. Journal of Electronics \& Information Technology, 2012, 31(12): 2953-2957. 